\title{
A NUMERICAL STUDY OF FLOW THROUGH WAVY-WALLED CHANNELS
}

\author{
S. SELVARAJAN ${ }^{\mathrm{a}}$, E.G. TULAPURKARA ${ }^{\mathrm{b}, *}$ AND V. VASANTA RAM ${ }^{\mathrm{c}}$ \\ "ExperimentaiAerodynamics Division, National Aerospace Laboratories, Bangalore 560 017, India \\ bepartment of Aerospace Engineering, Indian Institute of Technology, Madras 600 036, India \\ 'Institut fur Thermo- und Fluiddynamik, Ruhr-Universität Bochum, D.44780, Germany
}

\begin{abstract}
SUMMARY
A numerical procedure is developed for the analysis of flow in a channel whose walls describe a travelling wave motion. Following a perturbation method, the primitive variables are expanded in a series with the wall amplitude as the perturbation parameter. The boundary conditions are applied at the mean surface of the channel and the first-order perturbation quantities are calculated using the pseudospectral collocation method. Although limited by the linear analysis, the present approach is not restricted by the Reynolds number of the flow and the wave number and frequency of the wavy-walled channel. Using the computed wall shear stresses, the positions of flow separation and reattachment are determined. The variations in velocity and pressure with frequency of excitation are also presented. (C) 1998 John Wiley \& Sons, Ltd.
\end{abstract}

KEY WORDS: wavy channel flow; peristaltic motion; spectral collocation method

\section{INTRODUCTION}

Viscous flow over wavy boundaries has not been studied extensively although it is encountered in several phenomena, e.g. the generation of wind waves on water, the formation of sedimentary ripples in river channels and dunes in deserts, etc. Physiologists are interested in the study of blood and urinary flow in order to optimize artificial organs. The problem is worthy of interest because unsteady fluid dynamics such as this shows the development in time for viscous-inviscid interactions. There have been studies on flows in sinusoidally varying channels and pipes by Burns and Parkes [1] under the assumption that the Reynolds number is small enough for the Stokes approximation to be valid. They obtained a solution by expressing the streamfunction as a Fourier cosine series, determining the coefficients by assuming small values of the amplitude. Tsangaris and Leiter [2] improved the study by expressing the streamfunction as a Fourier series not in the physical plane but in a transformed plane where the wavy boundary is transformed into a straight one. They later extended the analysis to higher Reynolds numbers [3]. Steady and unsteady flows through furrowed channels have been investigated numerically by Sobey and co-workers [4,5], who studied the effect of Reynolds number and obtained a relation between the geometric parameters and the Reynolds number for which separation occurs. Fluid flow with and without heat transfer in wavy channels was calculated by Vajaravelu [6] using a perturbation method for long-wave channels.

* Correspondence to: Department of Aerospace Engineering, Indian Institute of Technology, Madras 600 036, India.

CCC 0271-2091/98/050519-13\$17.50

Received August 1996

(C) 1998 John Wiley \& Sons, Ltd.

Revised March 1997 
Although not directly related to the present work, it is interesting to note the studies on flows in tubes with constrictions. Young and Tsai [7] have investigated, theoretically and experimentally, the flow patterns in a stenosis (axisymmetric), also determining the Reynolds number effect on the flow separation. Some interesting experimental insight to the variation in pressure and shear stresses along a wavy pipe for turbulent non-separated flow was given by Hsu and Kennedy [8]. A numerical treatment of steady laminar separated flow in pipes with sinusoidal wall variation is presented by Chow and Soda [9]. The unsteady oscillatory viscous flow in pipes and channels of slowly varying cross-section has been calculated by Ramachandra Rao and Devanathan [10], Hall [11] and Cheng et al. [12].

Nishimura et al. $[13,14]$ investigated the characteristics of flow in a channel with symmetric wavy walls. In a flow regime ranging from laminar to turbulent, they studied the variation in pressure drop and wall shear stresses with Reynolds number. Recently Guzman and Amon [15] performed direct simulation in an effort to understand the evolution of transition from laminar to turbulent flow in a converging-diverging channel.

In the present work the shear flow in a channel whose walls are subjected to a wave-like forced excitation is studied using the pseudospectral collocation method. The formulation of the problem is presented in Section 2. This is followed by validation of the code and new results in Section 3.

\section{FORMULATION}

The non-dimensional forms of the equations of motion for an incompressible fluid in the usual notation are

$$
\begin{aligned}
& \frac{\partial u_{j}}{\partial x_{j}}=0, \quad 7=1,2,3, \\
& \frac{\hat{c} u_{j}}{\partial t}+t_{m} \frac{\hat{\partial} u_{j}}{c x_{m}}=-\frac{\partial p}{\partial x_{j}}+\frac{1}{R} \frac{\partial^{2} u_{j}}{\partial x_{m}} \partial, \quad j, m=1,2,3,
\end{aligned}
$$

where the lengths are non-dimensionalized w.r.t. the mean semi-channel height $H$, the velocity components w.r.t. the mean centreline velocity $U$, the time w.r.t. $H / U$ and the pressure w.r.t. $p U^{2}$. The Reynolds number $R$ is based on $H$ and $U$.

We specify the wall motion through the expressions (see Figure 1)

$$
x_{z_{\text {upper wall }}}=1-\epsilon_{k} \cdot \operatorname{Re}\left\{\exp \left[i\left(\hat{j}_{1} x_{1}+i_{3} x_{3}-\omega_{\mathrm{g}} t+\varphi_{\mathrm{p}}\right)\right\}\right\},
$$

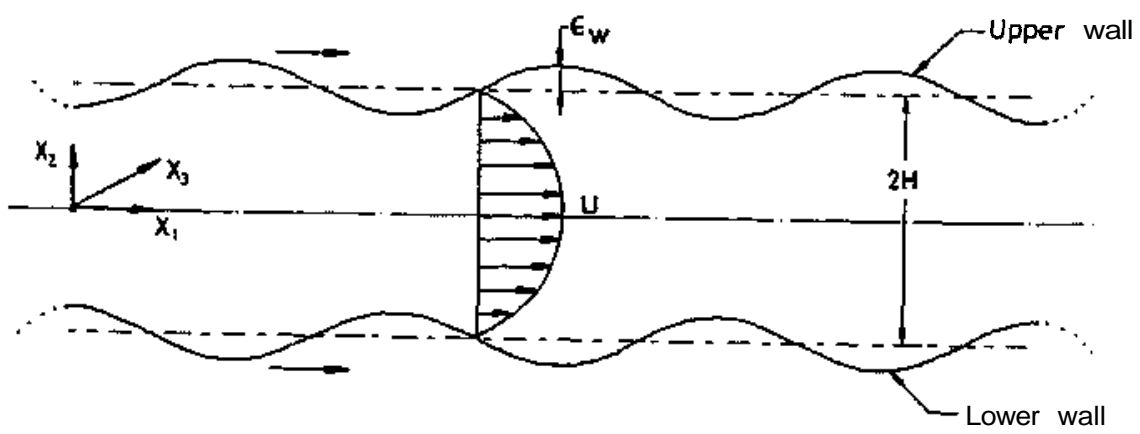

Figure 1. Flow configuration 


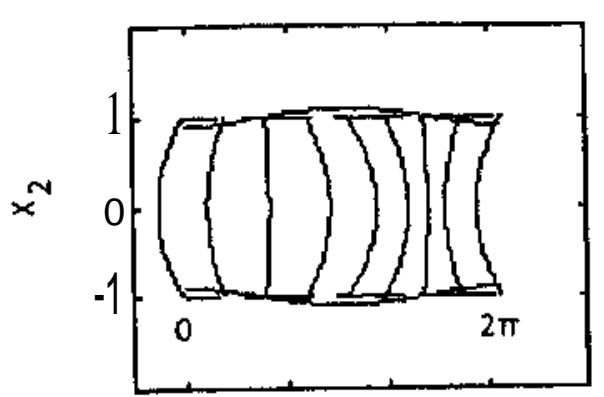

(a) $u_{i}$ profile

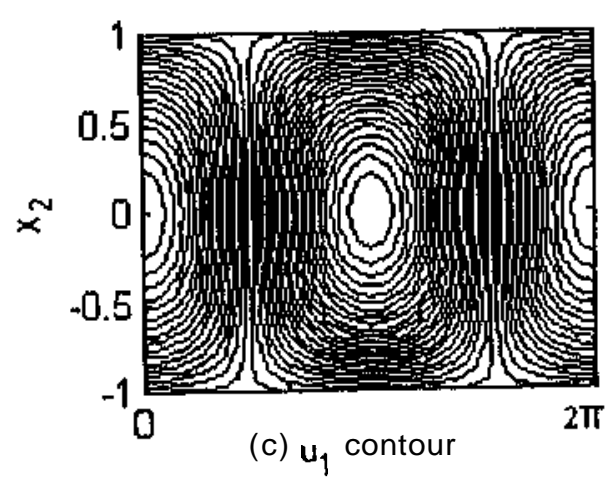

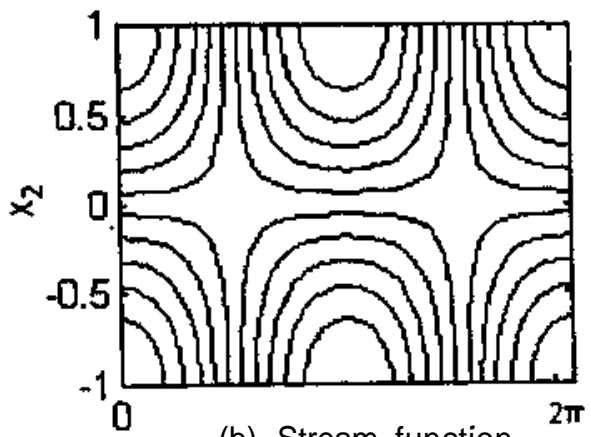

(b) Stream function

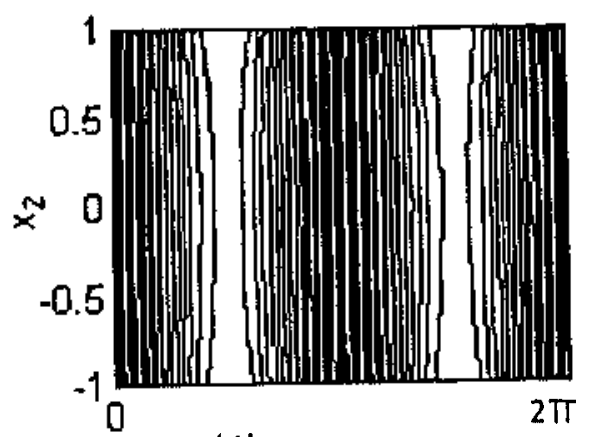

(d) $\mathrm{p}$ contour

Figure 2. Computation of peristaltic motion for $R=0.001, \varepsilon_{\mathrm{w}}=0.1, \lambda_{1}=0,25$ and $\omega_{\mathrm{g}}=4 \pi$

$$
x_{2_{\text {kwer wall }}}=-1+\varepsilon_{\mathrm{w}} \operatorname{Re}\left\{\exp \left[\mathrm{i}\left(\lambda_{1} x_{1}+\lambda_{3} x_{3}-\omega_{\mathrm{g}} t\right)\right]\right.
$$

which represent a wave travelling in the wall plane around the position $x_{2}= \pm 1$ with amplitude $\varepsilon_{\mathrm{w}}$, wave number vector $\left(\lambda_{1}, 0, \lambda_{3}\right)$ and frequency $\omega_{\mathrm{g}} ; \operatorname{Re}($.$) denotes the real part of$ (.). In the above form the waves of the upper and lower walls are in antiphase so that any instant of time the local channel width expands and contracts (symmetrically) around the value $2 H$. It is relatively simple to extend the study to pennit the waves on the two walls to be out of phase with each other (asymmetrically). It may be noted that an additional parameter, namely the phase difference $\varphi_{p}$, is included in (2a) for the sake of generality. The introduction

Table I. Variation in centreline velocity with Reynolds number for $\varepsilon_{\mathrm{w}}=0.1$,

\begin{tabular}{lrrrr}
\multicolumn{5}{c}{$\lambda_{1}=0.25$ and $\omega_{\text {g }}=4 \pi$} \\
\hline$x_{1}(\mathrm{rad})$ & $R=0.6$ & $R-0.06$ & $R=0.006$ & $R=0.001$ \\
\hline 0 & -0.1215 & -0.1277 & -0.1278 & -0.1278 \\
0.7854 & -0.0687 & -0.0832 & -0.0844 & -0.0845 \\
1.5708 & 0.0360 & 0.0217 & 0.0201 & 0.0199 \\
2.3562 & 0.1312 & 0.1256 & 0.1245 & 0.1244 \\
3.1416 & 0.1613 & 0.1675 & 0.1676 & 0.1676 \\
3.9270 & 0.1085 & 0.1230 & 0.1242 & 0.1243 \\
4.7124 & 0.0038 & 0.0181 & 0.0197 & 0.0199 \\
5.4974 & -0.0915 & -0.0858 & -0.0847 & -0.0846 \\
6.2832 & -0.1215 & -0.1277 & -0.1278 & -0.1278 \\
\hline
\end{tabular}




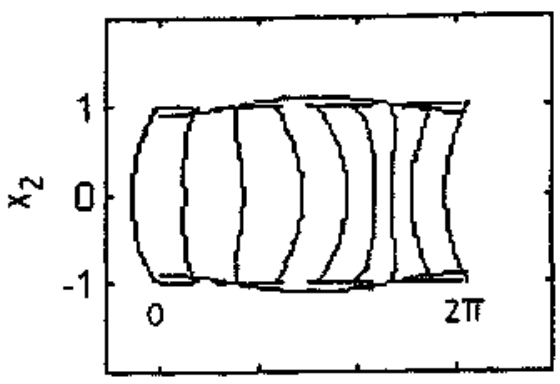

(a) u, protile

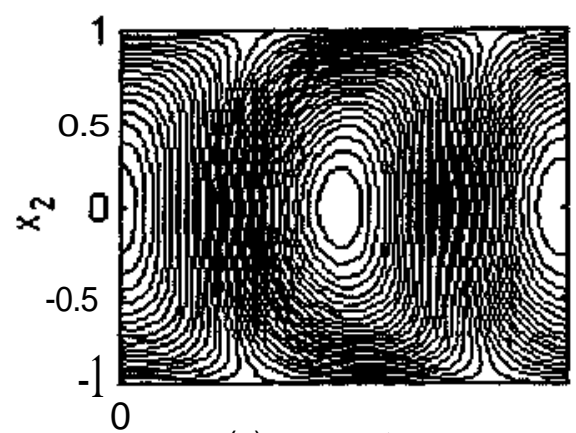

(c) $u_{1}$ contour

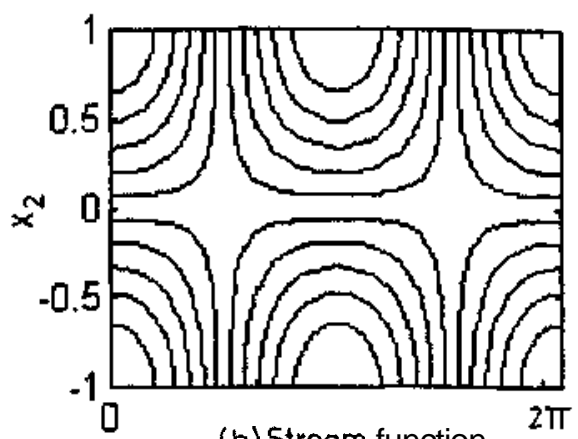

(b) Stream function

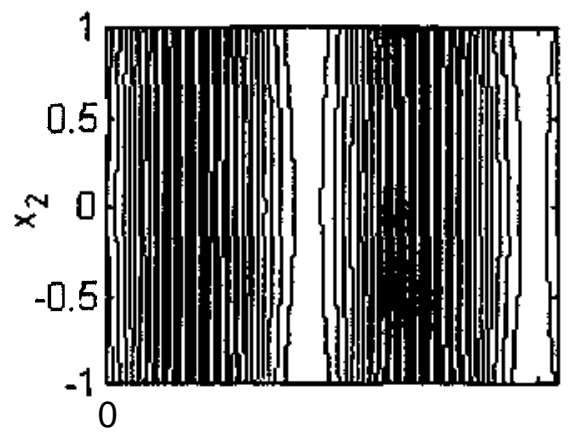

(d) $\mathrm{p}$ contour

Figure 3. Computation of peristaltic motion for $R=0.6, \varepsilon_{\nu_{v}}=0.1, \lambda_{1}=0.25$ and $\left(\nu_{\mathrm{g}}=4 \pi\right.$

of $\varphi_{p}$ would alter the basic flow significantly. Its role would be important in the study of the stability of the basic flow. In the present investigation, $\varphi_{\mathrm{p}}$ is taken to be zero.

For small amplitudes of wavy wall excitation the solution for the flow quantities may be sought as a perturbation from the parabolic velocity profile of the fully developed channel flow. To a linear approximation in $\varepsilon_{\mathrm{w}}$ we may then write the flow quantities as

$$
\begin{aligned}
& u_{t n}=\delta_{m \mathrm{~L}}\left(1-x_{2}^{2}\right)+\varepsilon_{\mathrm{w}} \tilde{u}_{m}+O\left(\varepsilon_{\mathrm{w}}^{2}\right), \quad m=1,2,3, \\
& p=-2 x_{\mathrm{l}} / R+\varepsilon_{\mathrm{x}} \bar{p}+O\left(\varepsilon_{\mathrm{w}}^{2}\right)
\end{aligned}
$$

where $\delta_{i j}$ is the Kronecker delta and $u_{\mathrm{i}}$ and $p$ are the periodic parts of the velocity components and pressure respectively.

Transferring the boundary conditions in $(2 \mathrm{a}, \mathrm{b})$ to the mean position of the wall by standard methods [16], we get the boundary conditions for the perturbation $u_{m}$ as

$$
\begin{aligned}
& \tilde{u}_{1}( \pm 1)=-2 \operatorname{Re}\left\{\exp \left[\mathrm{i}\left(\lambda_{1} x_{1}+\lambda_{3} x_{3}-\omega_{\mathrm{g}} t\right)\right]\right\} \\
& \tilde{u}_{2}( \pm 1)=\operatorname{Re}\left\{ \pm \mathrm{i} \omega_{\mathrm{g}} \exp \left[\mathrm{i}\left(\lambda_{1} x_{1}+\lambda_{3} x_{3}-\omega_{\mathrm{g}} t\right)\right]\right\} \\
& \tilde{u}_{3}( \pm 1)=0
\end{aligned}
$$

The form of the solution for $u_{m}$ may then be written as

$$
\tilde{u}_{m}=\frac{1}{2}\left\{\hat{u}_{m}\left(x_{2}\right) \exp \left[\mathrm{i}\left(\lambda_{1} x_{1}+\lambda_{3} x_{3}-\omega_{\mathrm{g}} t\right)\right]+\hat{u}_{m}^{*}\left(x_{2}\right) \exp \left[-\mathrm{i}\left(\lambda_{1} x_{1}+\lambda_{3} x_{3}-\omega_{\mathrm{g}} t\right)\right]\right\},
$$




$$
\tilde{p}=\frac{1}{2}\left\{\hat{p}\left(x_{2}\right) \exp \left[\mathrm{i}\left(\lambda_{1} x_{1}+\hat{\lambda}_{3} x_{3}-\omega_{\mathrm{g}} t\right)\right]+\hat{p}^{*}\left(x_{2}\right) \exp \left[-\mathrm{i}\left(\hat{\lambda}_{1} x_{1}+\lambda_{3} x_{3}-u_{\mathrm{g}} t\right)\right]\right\}
$$

where $u_{m}$ and $\hat{p}$ are the complex amplitude functions for velocities and pressure respectively; the superscript asterisk denotes the complex conjugate. Substituting Equations (5a, b) in (1a, b) and linearizing yields the following equations for $u_{m}$ and $\dot{p}$ :

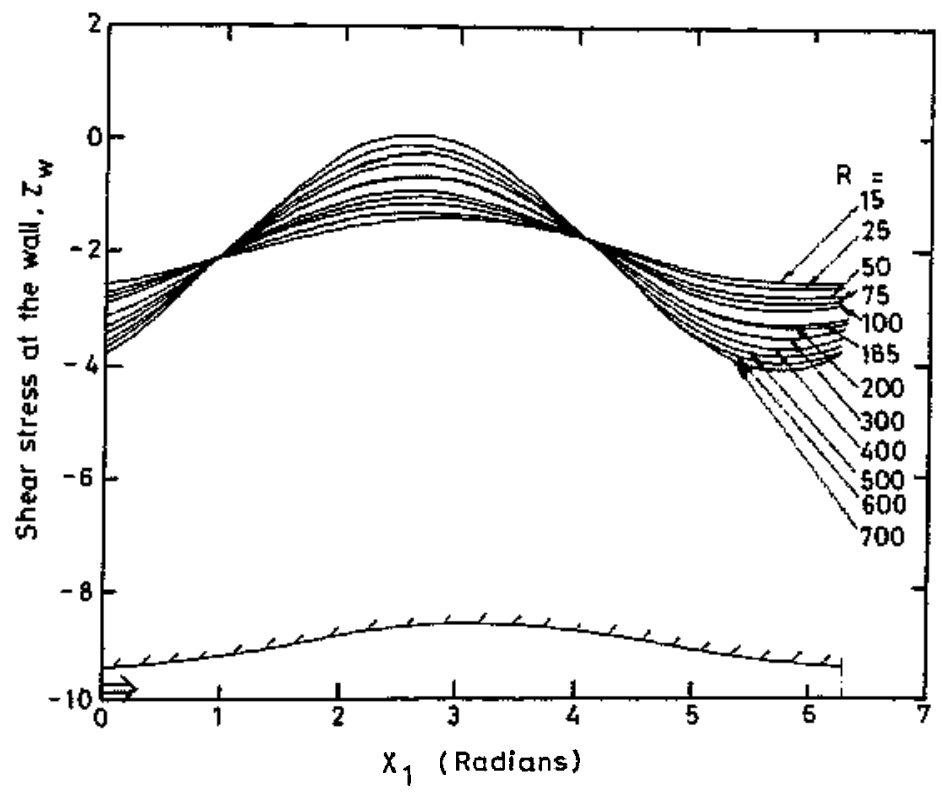

Figure 4. Variation in shear stress at wavy wall for $E_{w}=0.1$

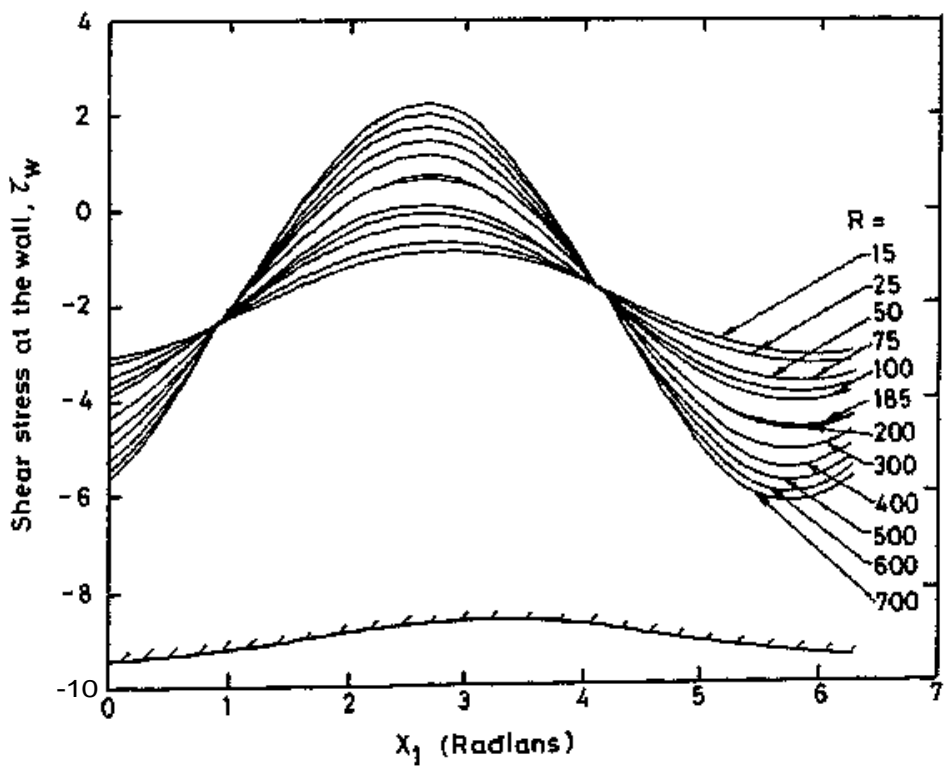

Figure 5. Variation in shear stress at wavy wall for $\varepsilon_{\mathrm{x}}=0.2$ 


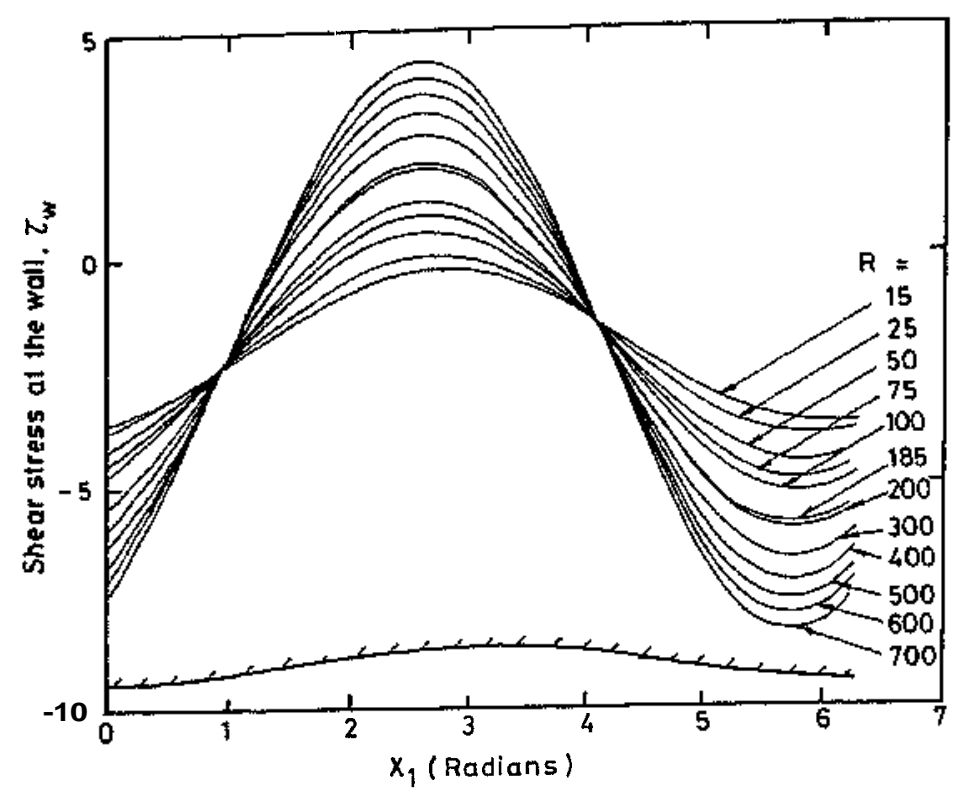

Figure 6. Variation in shear stress at wavy wall for $\varepsilon_{w}=0.3$

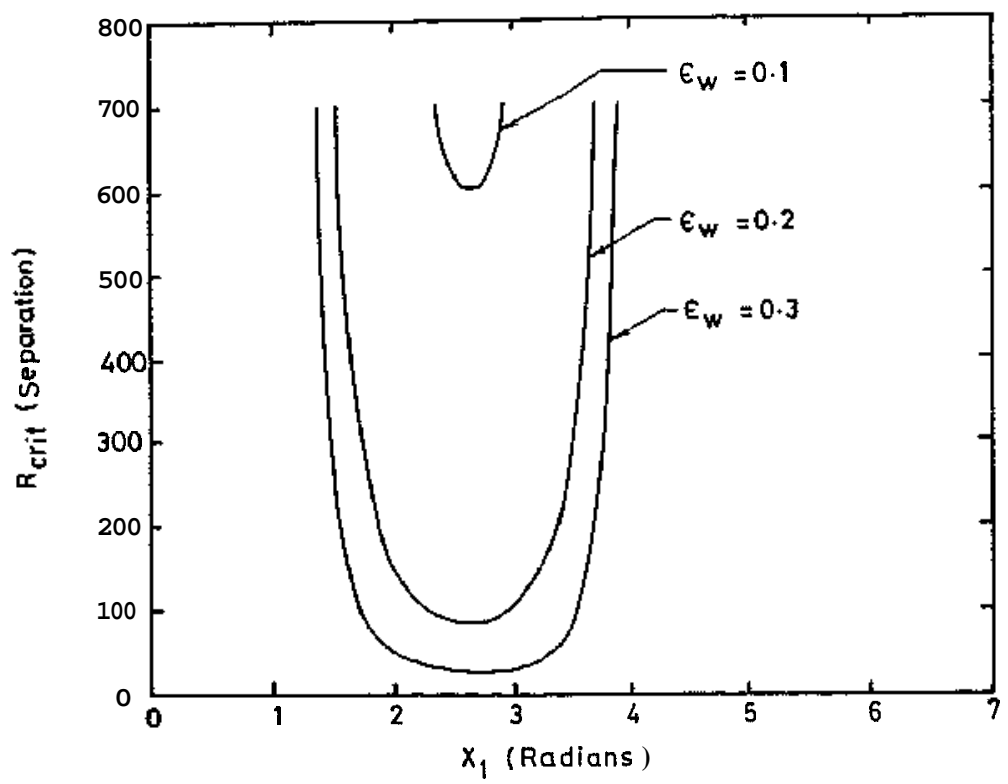

Figure 7. Variation in separation and reattachment points with Reynolds number

$$
\begin{aligned}
& \mathrm{i}\left[-\omega_{\mathbf{c}}+\left(1-x_{2}^{2}\right) \hat{\lambda}_{1}\right] \hat{u}_{1}-2 x_{2} \hat{u}_{2}=-\mathrm{i} \lambda_{1} \hat{\rho}+(1 / R)\left(-\lambda_{1}^{2}-\lambda_{3}^{2}+\quad \mathrm{rfVdxDW},\right. \\
& \mathrm{i}\left[-\omega_{\mathrm{g}}+\left(1-x_{2}^{2}\right) \lambda_{1}\right] \hat{u}_{2}=-\mathrm{d} \hat{p} / \mathrm{d} x_{2}+(1 / R)\left(-\lambda_{1}^{2}-\lambda_{3}^{2}+\mathrm{d}^{2} / \mathrm{d} x_{2}^{2}\right) \hat{u}_{2}, \\
& \mathrm{i}\left[-\omega_{\mathrm{g}}+\left(1-x_{2}^{2}\right) \lambda_{1}\right] \hat{u}_{3}=-\mathrm{i} \lambda_{3} \hat{p}+(1 / R)\left(-\lambda_{1}^{2}-\lambda_{3}^{2}+\mathrm{d}^{2} / \mathrm{d} x_{2}^{2}\right) \hat{u}_{3}, \\
& \mathrm{i} \lambda_{1} \hat{u}_{1}+\mathrm{d} \hat{u}_{2} / \mathrm{d} x_{2}+\mathrm{i} \lambda_{3} u_{3}=\mathbf{0} .
\end{aligned}
$$


Equations (6a-d) are the linearized equations of motion from which the well-known OrrSommerfeld equations for investigations of fluid flow stability can be derived [17,18]. The difference between the stability problem and the present investigation is the following inhomogeneous boundary conditions due to wall excitation:

$$
\mathbf{r f},( \pm \mathbf{l})=-\mathbf{2}, \quad \hat{u}_{2}( \pm 1)= \pm i \omega_{\mathrm{g}}, \quad \hat{u}_{3}( \pm 1)=0 .
$$

Equations (6a-d) along with the boundary conditions given by (7) are solved numerically by two methods. In the first method these equations are decomposed into a system of six first-order ordinary differential equations as follows. We define the variable $z_{j_{n}} j=1,2$, . ., 6 , as

$$
\mathrm{Z}_{2}=\hat{u}_{1}, \quad z_{2}=\dot{u}_{1}, \quad z_{3}=\hat{u}_{2}, \quad z_{4}=\hat{p}, \quad z_{5}=\hat{u}_{3}, \quad z_{6}=\dot{u}_{3},
$$

where the dot above the variable represents differentiation with respect to $x_{2}$. Then Equations $(6 a-d)$ can be written as

$$
\begin{aligned}
& \dot{\bar{Z}}=z_{2}, \\
& \dot{z}_{2}=\left[\mathrm{i} \lambda_{1} R\left(1-x_{2}^{2}\right)+\lambda_{1}^{2}+\lambda_{3}^{2}-\mathrm{i} \omega_{\mathrm{g}} R\right] z_{1}-2 x_{2} R z_{3}+\mathrm{i} \lambda_{1} R z_{4}, \\
& \dot{z}_{3}=-\mathrm{i} \lambda_{1} z_{1}-\mathrm{i} \lambda_{3} z_{5}, \\
& \dot{z}_{4}=(1 / R)\left[-\left(\lambda_{1}^{2}+\lambda_{3}^{2}\right)-\mathrm{i} \lambda_{1} R\left(1-x_{2}^{2}\right)+\mathrm{i} \omega_{\mathrm{g}} R\right] z_{3}-(1 / R) \mathrm{i} \lambda_{1} \dot{m}_{2}-(1 / R) \mathrm{i} \lambda_{3} z_{6}, \\
& \dot{z}_{5}=z_{6}, \\
& \dot{z}_{6}=\mathrm{i} \lambda_{3} R z_{4}+\left[\lambda_{1}^{2}+\lambda_{3}^{2}+\mathrm{i} \lambda_{1} R\left(1-x_{2}^{2}\right)-\mathrm{i} \omega_{\mathrm{g}} R\right] z_{5} .
\end{aligned}
$$

Equation (9) with the boundary conditions given by (7) are solved using the Scott and Watts [19] scheme. This involves a superposition coupled with an orthonormalization procedure with a variable step Runge-Kutta-Fernberg integration scheme. Each time the linearly independent solutions start to lose their numerical independence, the vectors are reorthonormalized before integration proceeds. The underlying principle of the algorithm is then to piece together the intermediate (orthoganalized) solutions, defined on the various subintervals, to obtain the desired solution. The details are given in Reference [20]. Sengupta [21] has also applied a similar procedure in the study of boundary layer flow over rigid and moving wavy surfaces using a wave-following co-ordinate system.

The second procedure uses the pseudospectral collocation method and is described by Canuto et al. [22]. The momentum and continuity Equations (6a-d) in matrix form appear as

$$
\left[\begin{array}{llll}
a_{11} & a_{12} & a_{13} & a_{14} \\
a_{21} & a_{22} & a_{23} & a_{24} \\
a_{31} & a_{32} & a_{33} & a_{34} \\
a_{41} & a_{42} & a_{43} & a_{44}
\end{array}\right]\left[\begin{array}{c}
\hat{u}_{1} \\
\hat{u}_{2} \\
\hat{u}_{3} \\
\hat{p}
\end{array}\right]=\left[\begin{array}{l}
0 \\
0 \\
0 \\
0
\end{array}\right],
$$

where the matrix coefficients $a_{i j}$ are given by

$$
\begin{aligned}
& a_{11}=a_{22}=\mathrm{fl}_{33}=D^{2}-\left(\lambda_{1}^{2}+\lambda_{3}^{2}\right)-\mathrm{i} \lambda_{1} R\left(1-x_{2}^{2}\right)+\mathrm{i} \omega_{g} R, \\
& a_{13}=a_{21}=a_{23}=a_{31}=a_{32}=a_{44}=0, \\
& a_{12}=2 R x_{2}, \quad a_{14}=-\mathrm{i} \lambda_{1} R, \quad a_{24}=-R D, \quad a_{34}=-\mathrm{i} \lambda_{3} R, \quad a_{41}=\mathrm{i} \lambda_{1},
\end{aligned}
$$




$$
a_{42}=D, \quad a_{43}=\mathrm{i} / 3,
$$

with $\mathrm{D}$ the differential operator matrix [22]. For a given number $n$ of collocation points distributed across the mean surfaces of the channel (from - 1 to 1), the matrix equations are formed using the differential operator matrix. The boundary conditions are applied by replacing the first and last rows of the first three equations in (10). The main advantage of the present method over similar perturbation methods, e.g. the streamfunction method of Sobey [4] Tsangaris and Leiter [2], is that the pressure and the phase information are obtained directly. For further details see Reference [20]. Both the finite difference method and the collocation method produced the same results for calculation of flow through a wavy channel at a Reynolds number of 150 .

Numerical experiments [20] indicated that as the Reynolds number is increased, the amplitude functions vary rapidly close to the boundaries. While using the finite difference method, sufficient points are needed for discretization of the domain closer to the wall. The collocation method allows the points to be distributed as a cosine function clustered close to the boundaries even for a moderate number of collocation points. Moreover, it retains all the spectral properties, namely the differential equations are solved exactly at the collocation points and the quantities are infinitely differentiable. Although not investigated in the present work, Canuto et al. [22] point out that the finite difference method may give rise to phase errors when compared with the collocation method. The main advantage of using the collocation method lies in the ease of computation with desired accuracies. The present work illustrates the use of the collocation method to solve a boundary value problem for which the finite difference method demands rigorous numerical treatments.

\section{NUMERICAL RESULTS}

Computations were done to simulate the results of Burns and Parkes [1] on peristaltic motion at low Reynolds numbers and those of Tsangaris and Leiter [3] at moderate Reynolds numbers. Various numbers of collocation points were taken across the channel. It was found that 41 points gave acceptably accurate results for the values of $R$ up to 8000 for which calculations were carried out. Orzag [23] and Brevdo [24] also recommended this number of points for finding the eigenvalues in the calculation of stability of channel flows.

\subsection{Development of asymmetry at low Reynolds numbers}

Burns and Parkes [1] observed that the velocity profiles are symmetric about the $x_{1}$-axis at all cross-sections. They also found that the values of $u_{1}$ are symmetric along the $x_{1}$-axis about the location $x_{1}=n$ (Figure $2 \mathrm{c}$ )). It may be recalled that they simplified the analysis by neglecting inertia terms in the equations. This symmetry was not observed by others [3] at low Reynolds numbers of order unity. The present formulation permits numerical solution for the peristaltic motion in the limit $R \rightarrow 0$ while retaining the inertial terms and could be used to examine this aspect. Computations at $R=0.6$ revealed the development of asymmetry. Hence computations were carried out for $R=0.06,0.006$ and 0.001 . Table I shows the numerical variation in $\boldsymbol{u}_{\mathbf{1}}$ along the centreline with Reynolds number $R$. It may be observed that the flow field gains symmetry as $R$ approaches the value of 0.001 , whereas a small value of 0.006 is sufficient to trigger asymmetry. Figures 2 and 3 show the velocity profile, streamfunction and velocity and pressure contours at $R=0.001$ and 0.6 respectively. The distortions due to asymmetry at $R=0.6$ are observed clearly in the velocity and pressure contours. 


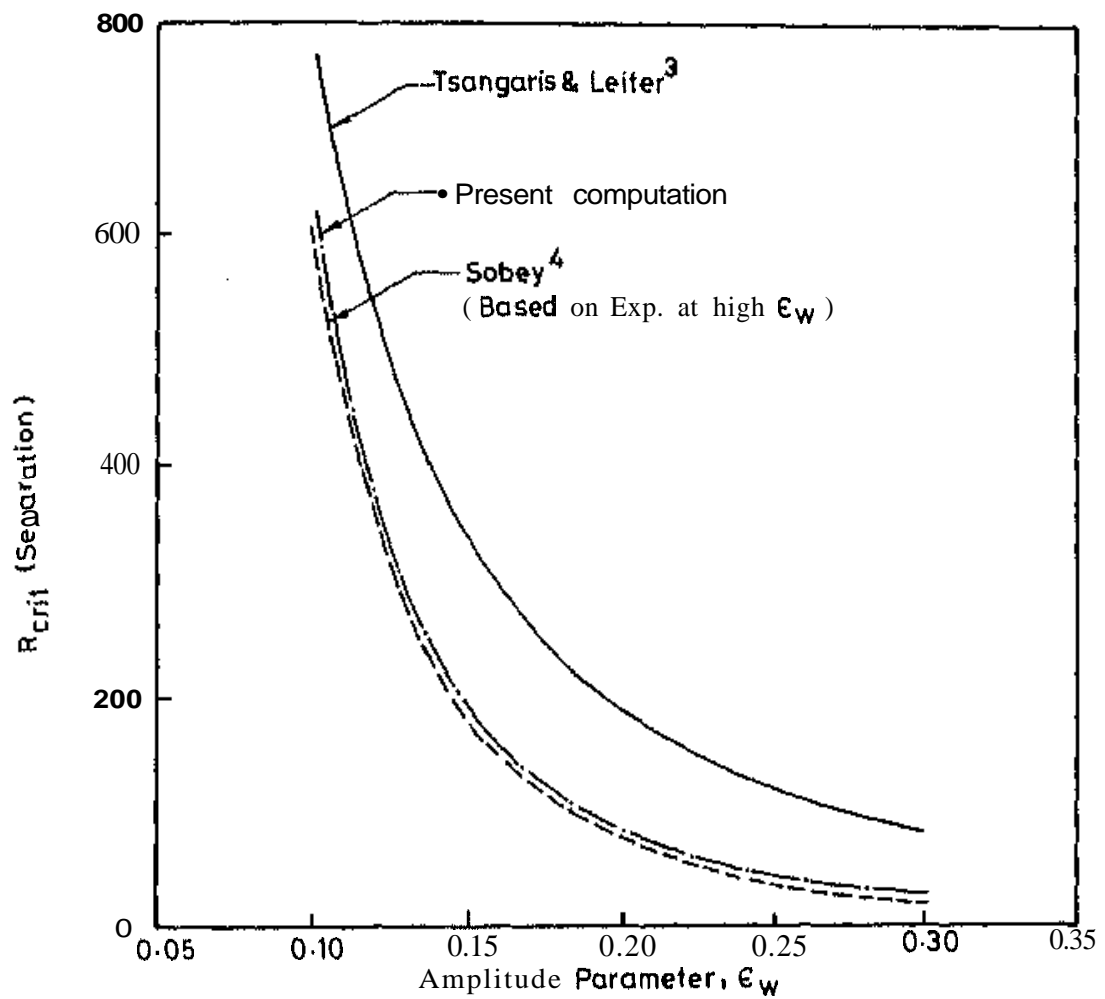

Figure X. Variation in $R_{\text {crit }}$ with amplitude parameter $\varepsilon_{w}$

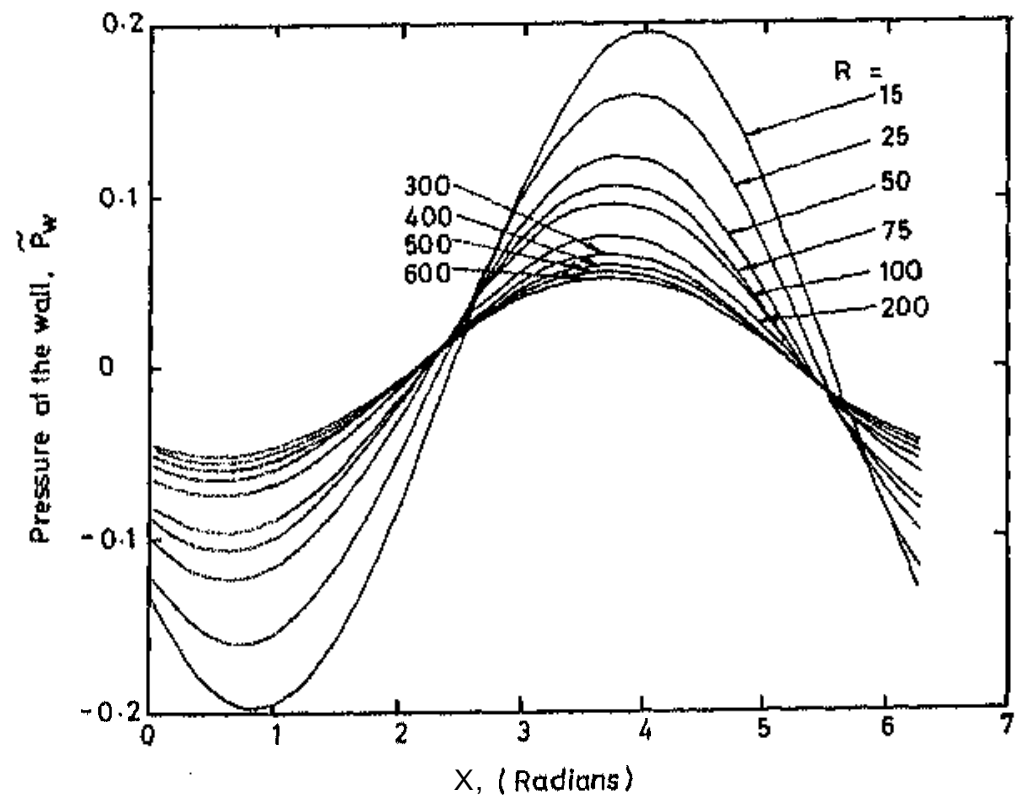

Figure 9, Variulion in pressure at wall for $c_{w}=0.2$ 


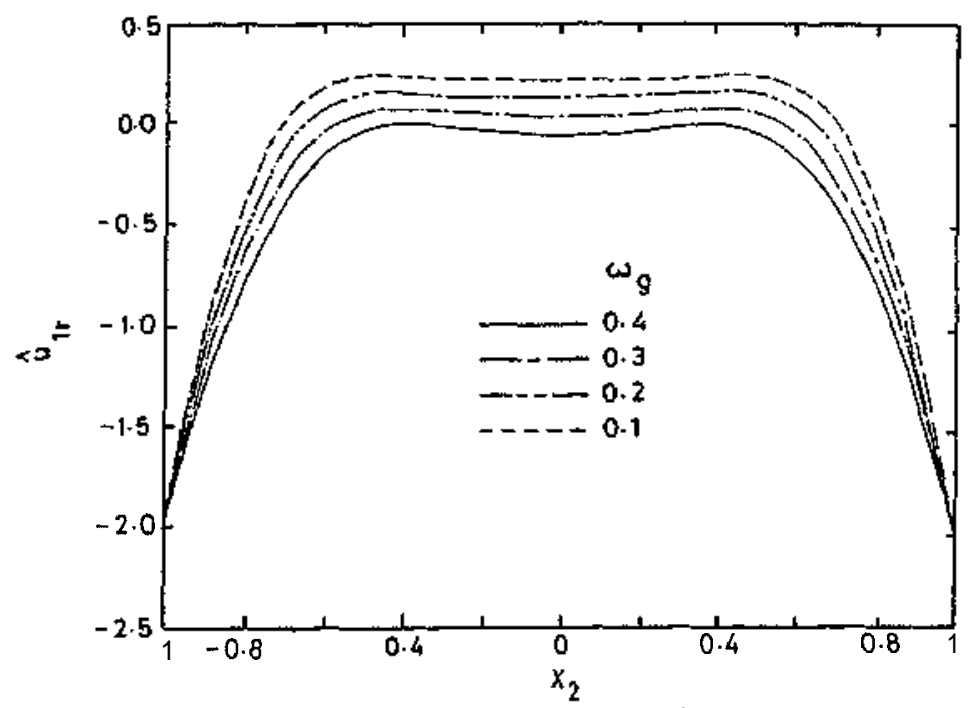

(a) Variation of $\hat{u}_{4 \mathrm{r}}$

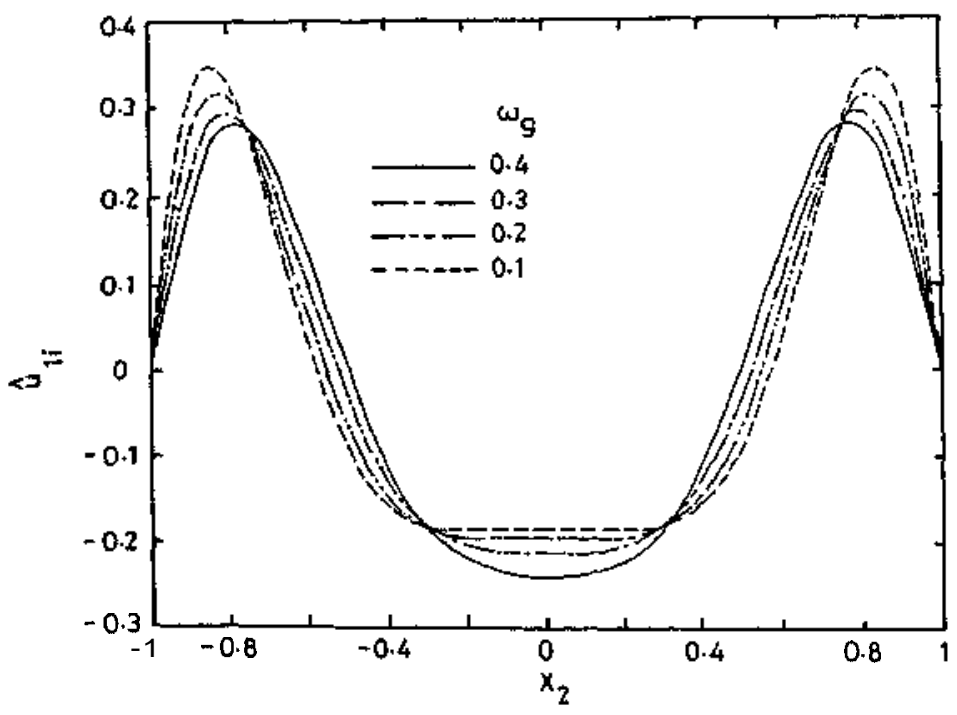

(b) Variation of $\hat{u}_{\mathrm{i}}$

Figure 10. Typical velocity amplitude functions for $R=120$ and $\lambda_{1}=1.058$

\subsection{Effect of $R$ and $\boldsymbol{\varepsilon}_{1 \mathrm{w}}$}

In this subsection we consider the effect of Reynolds number $R$ and amplitude parameter $\varepsilon_{\mathrm{w}}$ on the skin friction on a rigid wavy wall. This analysis is useful in itself and also provides locations of separation and reattachment, if any. Tsangaris and Leiter [3] have also carried out a similar analysis. Computations were carried out for $\varepsilon_{\mathrm{ww}}=0.1,0.2$ and 0.3 at $R=15,25,50$, $75,100,185,200,300,400,500,600$ and 700; $\lambda_{1}$ was taken as 1.0. Figures 4-6 show the variation in wall shear stress $\tau_{\mathrm{w}}$ along the boundary for $\varepsilon_{\mathrm{w}}=0.1,0.2$ and 0.3 respectively. It may be noted that the calculations are done between the mean surfaces of the channel. The 
wall shear stress $\tau_{\mathrm{w}}$, non-dimensionalized with $\rho U^{2}$, is obtained using the equation (Vajaravelu [6] also followed the same procedure)

$$
\tau_{\text {w|wavy wall }}=\left.\frac{\mathrm{d} u_{1}}{\mathrm{~d} x_{2}}\right|_{\text {wavy wall }}=\left.\frac{\mathrm{d} u_{0}}{\mathrm{~d} x_{2}}\right|_{ \pm i}+\left.\hat{\kappa}_{\mathrm{w}} \frac{\partial \tilde{u}_{1}}{\partial x_{2}}\right|_{ \pm 1}+\left.\varepsilon_{\mathrm{w}} \frac{\mathrm{d}^{2} u_{0}}{\mathrm{~d} x_{2}^{2}}\right|_{ \pm 1} \mathrm{e}^{\mathrm{i}\left(\hat{\lambda}_{1}, x_{1}+\lambda_{3} x_{3}-\varepsilon_{y_{g}}\right)}
$$

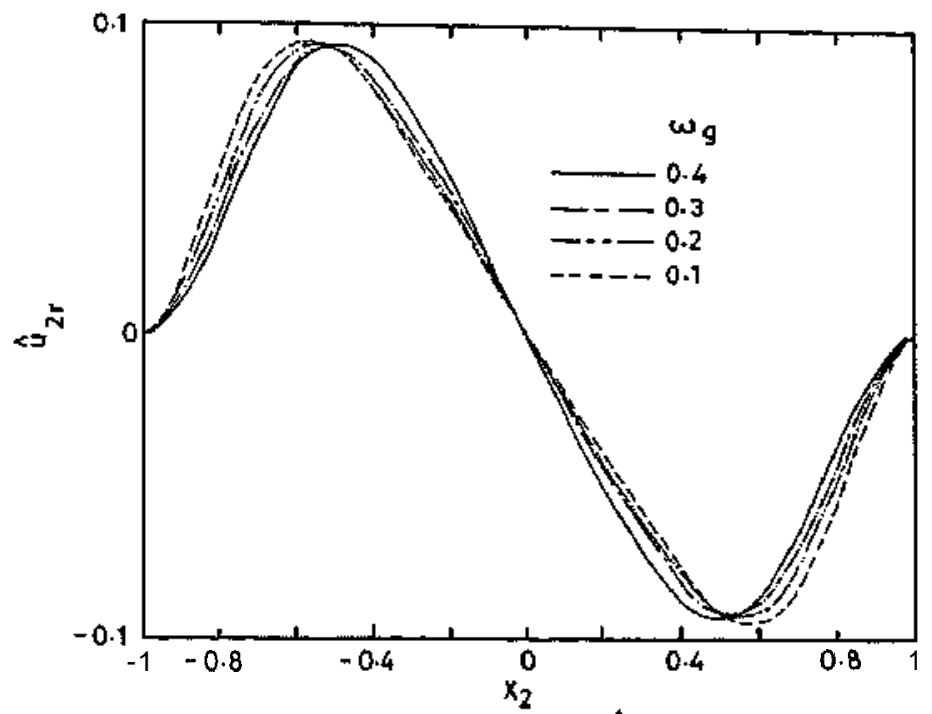

(c) Variation of $\hat{\mathbf{u}}_{2 r}$

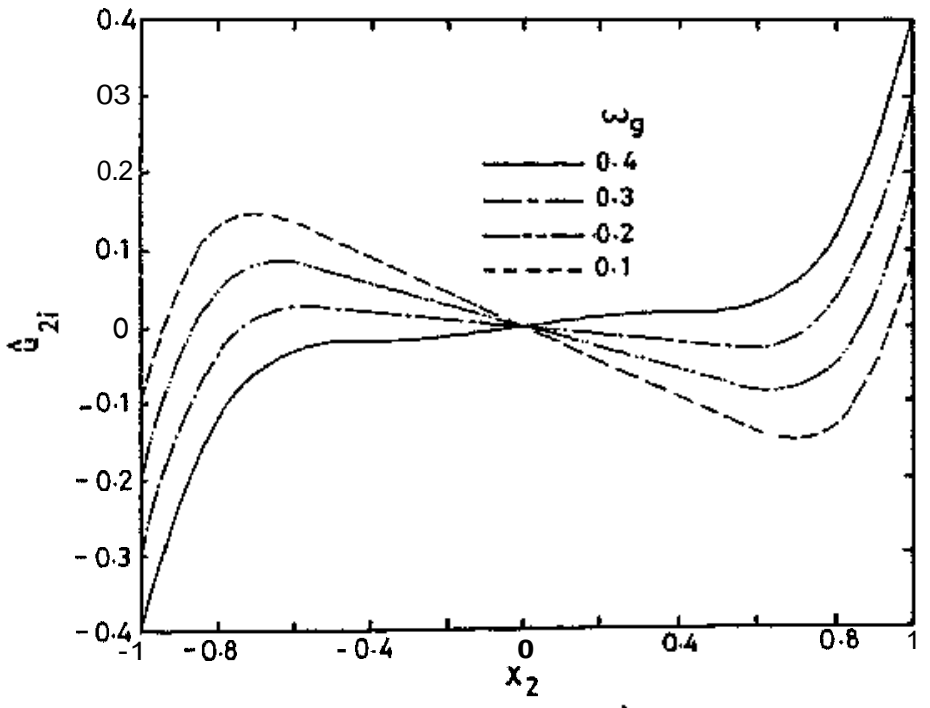

(d) Variation of $\hat{u}_{k i}$

Figure 10 (Continued) 
where $u_{0}=1-x^{2}$. It may be observed from Figures 4-6 that the variation in shear stress is a periodic function of $x_{1}$ having a phase difference with the variation in wall shape. Its maximum value lies upstream of the maximum channel cross-section. The amplitude of shear stress along the wall increases with increase in Reynolds number. Since the flow is two-dimensional and the wall is rigid, the separation and reattachment points are the locations where the shear stress is zero. (Separation on a moving boundary would require satisfaction of the MRS criterion [25].) For $\varepsilon_{w}=0.1$ the shear stress variation is negative and the flow is attached fo'r Reynolds numbers below 600. For $R=700$ a separated flow region on the channel wall exists. For $\varepsilon_{\mathrm{x}}=0.2$ the flow is attached only when $R$ is less than about 150 . The value of Reynolds number for which the flow just separates is called the 'critical Reynolds number' [3]. Above this critical Reynolds number a separation bubble exists. The extent of the bubble increases as $R$ increases. For $\varepsilon_{\mathrm{wv}}=0.3$ the critical Reynolds number is about 25 as shown in Figure 6. Figure 7 shows the positions of separation and reattachment points for $\varepsilon_{\mathrm{w}}=0.1,0.2$ and 0.3 for the wavy channel. The variation in critical Reynolds number with amplitude parameter is shown in Figure 8. The variation compares well with the correlation obtained using the experimental data of Sobey [4]. The results of Tsangaris and Leiter [3] are also plotted in the figure. The agreement between the experimental data and the present results may be attributed to the fact that there are no approximations involved in the formulation, except for linearization, and accurate solutions have been obtained using the spectral collocation method.

\subsection{Variations in wall pressure and velocity with frequency}

In the present formulation the oscillatory pressures are computed directly. The variations in oscillatory pressure at the wall along $x_{1}$ for $\varepsilon_{\mathrm{w}}=0.2$ and $R=15,25,50,75,100,200,300,400$, 500 and 600 are shown in Figure 9. It is interesting to note that the amplitude of pressure decreases with increase in Reynolds number. It follows from the formulation that the velocity amplitude functions $\boldsymbol{u}_{1}$ and $\boldsymbol{u}_{2}$ are complex quantities and for the sake of completeness the real and imaginary parts of $u_{1}$ and $u_{2}$ across the channel $\left(\hat{u}_{1 \mathrm{r}}, u_{1 \mathrm{i}} ; u_{2 \mathrm{r}}, \hat{u}_{2 \mathrm{i}}\right)$ are shown in Figures 10(a) -10 (d) with $\omega_{\mathrm{g}}$ as parameter. The chosen value of $R$ lies in the Reynolds number range of blood flows in arteries.

\section{CONCLUSIONS}

In the present investigation, using a simple perturbation approach and the collocation method, the incompressible flow through a wavy-walled channel is studied numerically. The evolution of flow asymmetry in a peristaltic motion even for a very low $R=0.006$ is captured. Although limited by the linear analysis, the present procedure is a useful tool for analysis of complex unsteady flow in a wavy channel. The separation criterion relating the critical Reynolds number $R_{\text {crit }}$ and the amplitude parameter $\varepsilon_{\mathrm{w}}$ as obtained by the present method is in conformity with other known methods. Further, the numerical procedure allows a variety of basic unsteady periodic flows to be computed for which the stability characteristics may be of interest.

\section{ACKNOWLEDGEMENTS}

The authors would like to thank Dr. T.K. Sengupta, Indian Institute of Technology, Kanpur and Mr. G. Rajendra, National Aerospace Laboratories, Bangalore for their valuable suggestions during the course of the present work. 


\section{REFERENCES}

1, J.C. Burns and T. Parkes, 'Peristaltic motion', J, Fluid Meth, 29, 731-743 (1967).

2. S. Tsangaris and E. Leiter, 'Analytical solution for weakly-stenosed channels at low Reynolds numbers'. Pros, lst Int. Corff: on Mechanics in Mi dirine and Biology, 1978, pp. 289--292.

3, S. Tsangaris, E. Leiter, 'On laminar steady flow in sinusoidal channels', J. Engerg. Made 18, 89-103 (1984).

4. 1.3. Sobey,'On flow through furrowed channels, Part 1: Calculated flow patterns', J. Fluid Meeh., 96, 1 -26 [1980).

5. K.D. Stephanhoff, 1.J. Sobey and B,J. Bellhouse, 'On flow through furrowed channels, Part 2: Observed flow patterns', J. Fhtid Mesas., 95, 27--32 (1980).

6. K. Vajaravelu, 'Fluid flow and heat transfer in horizontal wavy channels', Acia Mech., ${ }^{35,}$ 245--258 (1980).

7. D.F. Young and F.Y. Tsai, 'Flow characteristics in models of arterial stenosis. f. Steady flow, 11. Unsteady flow', J. Biotech., 6, 395-410, 547-559 (1973).

8. S.T. Hsu and J.F. Kennedy, 'Turbulent flow in wavy pipes', J. Fiiid Mech., 47, 481-502 (1972).

9. J.C.F. Chow and K. Soda, 'Laminar flow in tubes with constriction', Pltys. Fluids, 15, $1700-1706$ (1911).

10. A. Ramachandra Rao and R, Devanathan, 'Pulsatile flow in tubes of varying cross-section', ZAMP, 24, 203 -213 (1973).

11. P. Hall, 'Unsteady viscous flow in a pipe of slowly varying cross-section', J. Fluid Mech., 64, 209-230 (1974).

12. L.C. Cheng, M,E. Clark and W.C. Peng, 'The effect of oscillatory frequency in plane wavy conduit flows', J. Bianrec h., 10, 607-609 (1977).

13. T. Nishimura, Y. Ohasi and Y. Kawamura, 'Flow characteristics in a channel with symmetric wavy wall for steady flow', J. Chem. Engug. Jim., 17, 466-471 (1934).

14. T. Niishimura, S. Murakami, S. Ar,kawa, Y, Kawamura, 'Flow observations and mass transfer characteristics to symmetrical, wavy walled channels at moderate Reynolds numbers for steady now', Let. J. (fear Mass Transfer, 33, 835--845 (1990).

15. A.M. Guzman and C.H. Amon, 'Transition to chaos in converging-diverging channel flows: Ruelle-TakensNewhouse scenario', PhDs. Fluids, 6, 1994-2002 (1994).

16. M. van Dyke, Perturhcitiwi Methods in Fluid Mechanics, Parabolic, Stanford, CA. 1975.

17. C.C. Lin, The Theory rf'Nvdrodvricunfr Stability, Cambridge University Press, Cambridge, 1955.

18. P.G. Drazin and W.H. Reid, Hydrodynamic Stability, Cambridge University Press, Cambridge, 1981.

19, M. R. Scott and H.A. Waits, 'Computational solution of linear two-point boundary value problems via orthonormaliztttion', SIAM J. Nurner. Anal., 14, 40-70 (1977).

20. S. Selvarajan and V. Vasanta Ram, 'Dynamical characteristics of wave-excited channel flows', Bericht 162, Institut fiir Thermo. and Fiuiddynamik, Ruhr-Universiti t Bochum, 1991.

21. T. K. Se n gupta, 'To rbulent boundary layers over rigid and moving wavy surfaces', Ph.D. Thesis, Georgia Institute of Technology, 1984.

22. C. Canuto. M.Y. flussaini, A. Quarteroni, T.A. Zang, Spectral Methods in Fluid Dynarnies, Springer, Heidelberg, 1988.

23. S. A, Orzag, 'Accurate solution of the Orr-Sommerfeld stability equation', J. Fluid Mech., 50, 689-703 (197i).

24, L. f3revdo, 'Spatially amplifying waves waves in plane Poiseuille flow', ZAMM, 12, 163-174 (1992).

25. D.P. Telionis, Unsteady Flows, Springer, New York, 198). 